\title{
Depresión, discapacidad y sus costes. Propuestas de mejora de asistencia en Atención Primaria
}

\section{Depression, disability and its costs. Proposals to improve assistance in Primary Care}

Fecha de recepción: 14-03-2018

Fecha de aceptación: 15-06-2018
Jesús Alberto Santolaya Prego de Oliver

Universitat de València

María Modrego

Universidad Católica de Valencia

Gema Aonso

Universidad de Oviedo

Leonardo Adrían Medrano

Universidad Siglo XXI

Roger Muñoz

Universitat de València

\section{resumen/alsstract:}

Los trastornos emocionales y en especial, la depresión, son los trastornos más prevalentes tanto a nivel global como en España. Estos trastornos ocasionan grandes costes a nivel personal como social, generando una elevada discapacidad y dependencia. El objetivo de este trabajo es revisar el estado de la cuestión en cuanto al impacto de la depresión a nivel económico, así como de otros trastornos mentales comunes. Se esclarecerá la situación actual de los costes asociados a los trastornos del cerebro (trastornos neurológicos y trastornos mentales), específicamente los asociados a los trastornos emocionales del rango de la depresión, ansiedad y las somatizaciones. Además, se relacionarán con algunos factores que podrían estar condicionando la asistencia recibida en el sistema de salud español, describiendo algunas posibles deficiencias que de ser corregidas mejorarían la atención de los pacientes que presentan alguno de estos trastornos mentales comunes, mejorando así la gestión de los recursos disponibles con lo que repercutiría en un decremento de los costes asociados.

Emotional disorders and, in particular, depression, are the most prevalent mental disorders, as at global and also at Spain. These disorders cause to great personal, economical and social costs, generating high disability and dependence. The objective of this paper is to review the state of the issue regarding the impact of depression at the economic level, as well as other common mental disorders. Will be clarify the current situation of the economical costs associated with brain disorders (neurological disorders and mental disorders), specifically those associated with emotional disorders of the range of depression, anxiety and somatization. In addition, will be related to some factors that could be conditioning the assistance received in the Spanish health system, describing some possible deficiencies that, if corrected, would improve the care of patients who present some of these common mental disorders, improving the management of available resources with what would result in a decrease in associated costs.

\section{palabras clave/keywords:}

Depresión, Ansiedad, Somatizaciones, Discapacidad, carga económica.

Depression, Anxiety, Somatizations, Disability, Primary Care, Economic cost. 


\section{Introducción}

El estado de ánimo depresivo es una de las condiciones que más frecuentemente se encuentra en diferentes servicios sanitarios como, por ejemplo, en Atención Primaria (AP) (Roca et al., 2009). Algunos autores, como Santos-Ruiz, Sanz-Rodríguez y Álvarez-Calderón (2014) se aproximan al estudio de los trastornos del estado de ánimo como síndromes compuestos por síntomas de diferentes niveles. Así entonces, las manifestaciones de síndromes depresivos se componen síntomas anímicos, motivacionales, de conducta, cognitivos, físicos e interpersonales, que se describen a continuación.

Dentro de los síntomas anímicos, aparece tristeza e irritabilidad, acompañadas también con sensaciones de abatimiento, vacío y angustia. Dentro de los síntomas motivacionales y conductuales encontramos la triple A, es decir; la abulia, que consiste en la incapacidad de llevar a cabo los deseos que se admiten como elementales por el sujeto; la apatía, caracterizada por la falta de motivación, que no se haya relacionada con estrés, déficit intelectual o estados alterados de conciencia y que puede ser un estado extremo de la abulia y, finalmente; la anhedonia, que consiste en la pérdida o ausencia de la capacidad para experimentar placer físico o con la falta de interés relacionado con las situaciones que implican interacción con pares. A nivel conductual se haya en los pacientes con sintomatología del ánimo depresiva tanto un enlentecimiento generalizado del habla como en las respuestas motoras o gestuales. A ello se le ha de sumar síntomas físicos, como los relativos al apetito, el sueño, molestias corporales o cambios en el deseo sexual. Finalmente, en la esfera cognitiva, se encuentra una disminución en las capacidades cognitivas, tanto en las automáticas como aquellas que necesiten un control ejecutivo y esfuerzo más amplios. A parte del rendimiento, en las tareas que requieren del uso de estos procesos, también encontramos, en cuanto al contenido de las cogniciones, distorsiones sobre el entorno, el pasado y el futuro, así como pensamientos de autodesprecio, culpa o muerte.

En lo referente a la clasificación de las formas de depresión, dentro del Diagnostic and Statistical Manual of Mental Disorders, en su cuarta edición revisada, (DSM-IV-TR; APA, 2000), los trastornos depresivos se incluyen en el capítulo "Trastornos del Estado de áni$m o "$. Dicha clasificación diferencia entre trastornos basados en la etiología (que serán aquellos debidos a enfermedad médica o inducidos por sustancias), trastornos bipolares (Trastorno Bipolar I; Trastorno Bipolar II; Trastorno Ciclotímico; Trastorno Bipolar no especificado) y trastornos unipolares (trastorno depresivo mayor o TDM; trastorno distímico y; trastorno depresivo no especificado). Por supuesto también incluye la descripción de los episodios en los que se basan cada uno de estos trastornos.

Centrándonos en el apartado de los trastornos unipolares, los criterios para el episodio depresivo mayor versan sobre la presencia de 5 o más síntomas de los cuales, entre otros, debe aparecer necesariamente la incapacidad para experimentar placer o el estado de ánimo depresivo, además de variaciones en el peso importantes, insomnio o hipersomnia, agitación o enlentecimiento psicomotor, fatiga, pensamientos recurrentes de muerte, disminución de la capacidad para pensar o concentrarse y sentimientos de inutilidad. La sintomatología no 
ha de cumplir criterios para un episodio mixto y ha de provocar interferencia en funcionamiento del individuo, no pudiéndose achacar a una enfermedad médica, al efecto de alguna sustancia o a la presencia de duelo (APA, 2000). Para el trastorno depresivo mayor (TDM), esta condición supondrá la presencia de un único episodio que no se explica mejor por otros trastornos en el que nunca se ha producido episodios maniaco, hipomaniaco o mixto (salvo si están inducidos por sustancias o condición fisiológica), en el que se especifica su gravedad (leve, moderado, grave sin síntomas psicóticos, grave con síntomas psicóticos, en remisión parcial/ total o sin especificar) y si es crónico o se presenta con sintomatología catatónica, melancólica, atípica o de inicio en el posparto. (DSM-IV-TR, APA, 2000). En la última versión recientemente publicada del DSM-5, estos criterios se han mantenido en mayor medida en el TDM. Tan solo se han dado pocos cambios que se refieren a la inclusión de dos categorías que acotan la ideación suicida: el desorden del comportamiento suicida y la autolesión no suicida. Además, también se incluye el trastorno depresivo persistente, como una unión del trastorno distímico y el depresivo mayor crónico.

El TDM se ha convertido en los últimos años en uno de los trastornos psicológicos más prevalentes en la población general y uno de los más incapacitantes en todo el mundo (Paykel, Brugha, \& Fryers, 2005). Mathers \& Loncar, (2006) predicen que la depresión, se convertirá en una de las primeras causas de discapacidad llegado el año 2030. Asimismo, en España es también un trastorno muy prevalente. En un estudio realizado por Haro y colaboradores en 2006 se evaluó la epidemiologia de los trastornos mentales en población española, estimando que un $15,7 \%$ de los hombres y un 22,9\% de las mujeres de nacionalidad española sufrían algún trastorno mental a lo largo de su vida (Figura 1). En relación a la incidencia anual, el 8,4\% de la población (un 5,2\% para el sexo masculino y un $11,4 \%$ para el femenino) habían presentado algún diagnóstico en el año previo a la elaboración del estudio (Haro et al., 2006). La distribución ponderada de los principales trastornos mentales en población española es la siguiente; para los trastornos del estado de ánimo un 4,37\% en prevalencia año y un 11,47\% prevalencia vida; para los trastornos de ansiedad un 7,61\% en prevalencia año y un $9,39 \%$ prevalencia vida; para los trastornos por consumo de alcohol un $0,69 \%$ en prevalencia año y un 3,60 \% prevalencia vida; para cualquier trastorno mental un $8,48 \%$ en prevalencia año y un $19,46 \%$ prevalencia vida. Dentro de los trastornos de estado de ánimo, el TDM es el más frecuente con 3,9 de prevalencia-año y un 10,5 de prevalenciavida. Asimismo, según este estudio (Haro et al., 2006), los trastornos mentales, entre ellos la depresión, ocasionaron el mayor impacto negativo sobre la calidad de vida relacionada con la salud y la discapacidad funcional (más días de trabajo perdidos), superando incluso el impacto de enfermedades físicas crónicas. Estos datos muestran la envergadura del problema al que nos enfrentamos. Por tanto, este trabajo pretende realizar una revisión del estado de la cuestión que envuelve a la depresión, uno de los trastornos más incapacitantes (Ruiz-Rodríguez et al., 2017) y el estudio de sus costes asociados. Finalmente, se revisará qué impacto tiene en atención primaria (AP) y qué medidas pueden proponerse para hacer frente a esta situación. 


\section{Estado de la cuestión}

\section{Discapacidad y depresión}

El núcleo esencial de la discapacidad es un estado de interferencia de funcionamiento tanto a nivel intra-personal como inter-personal, afectando a diferentes esferas de la vida y que viene derivado del padecimiento de una merma en las funciones físicas, sensoriales o psíquicas que serían consideradas típicas para un sujeto de la misma edad.

Figura 1: Prevalencia de los trastornos mentales en España a lo largo de la vida. Fuente: Elaboración propia a partir de Haro et al (2006)

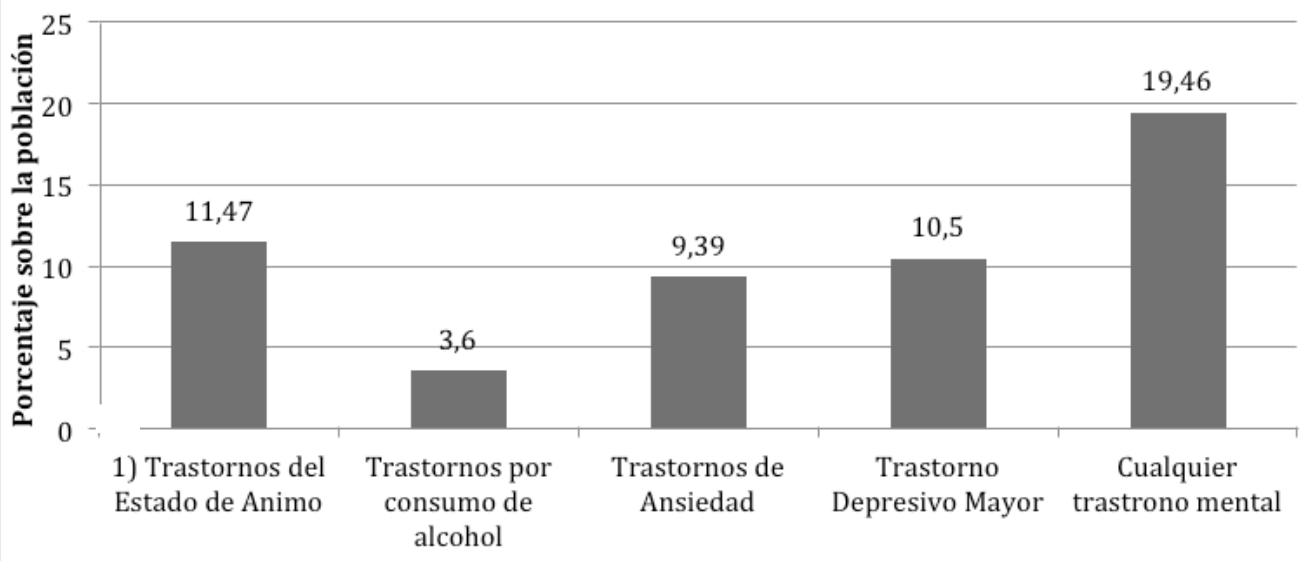

Según la última encuesta EDAD (Encuesta de Discapacidad, Autonomía Personal y Situaciones de Dependencia; MSSS, 2008), del Instituto Nacional de Estadística (INE), realizada sobre un total de 2.710 .000 personas, se observa que una media de $8,5 \%$ de la población sufre algún tipo de discapacidad, siendo un $6,9 \%$ en varones y un $10,1 \%$ en mujeres (INE, 2008). La distribución por edad y sexo de las personas con dependencia en España es de un $59.7 \%$ mujeres y un $40,3 \%$ varones. De estos, el 2,8\% son menores de 6 años, un 2,2\% se encuentran entre 6 y 15 años, el $28,8 \%$ entre 16 y 64 , mientras que encontramos un $65,2 \%$ en población mayor de 65 años.

Asimismo, en la encuesta EDAD también se profundiza en las causas de la discapacidad (Figura 2), estimando que la distribución de las personas con un grado de dependencia superior al 33\% se asignaba a los siguientes grupos de enfermedades: enfermedades osteoarticulares, 28,05\%; enfermedades crónicas 18,01\%; enfermedades mentales 16,06\%; déficit intelectual, 8,96\%; déficit visual, 7\%; déficits auditivos, 5,79\%; mixtos, 2,67\% y finalmente; otros, $2,31 \%$. Estos datos muestran que el perfil de la persona con discapacidad es principalmente femenino, siendo aproximadamente dos tercios de la población con discapacidad mayores de 65 años. Dentro de estas, las personas con una discapacidad cuya causa no deriva de otro tipo de alteraciones, (es decir, constan como primera causa los problemas de salud mental) y que además tienen reconocido un mínimo de un $33 \%$ de discapacidad 
Figura 2: Causas de discapacidad y porcentaje de personas con una discapacidad superior al 33\%. Fuente: Elaboración propia a partir del Informe a 31/12/2015 de la base estatal de datos de personas con valoración del grado de discapacidad de la Subdirección general de planificación, ordenación y evaluación.
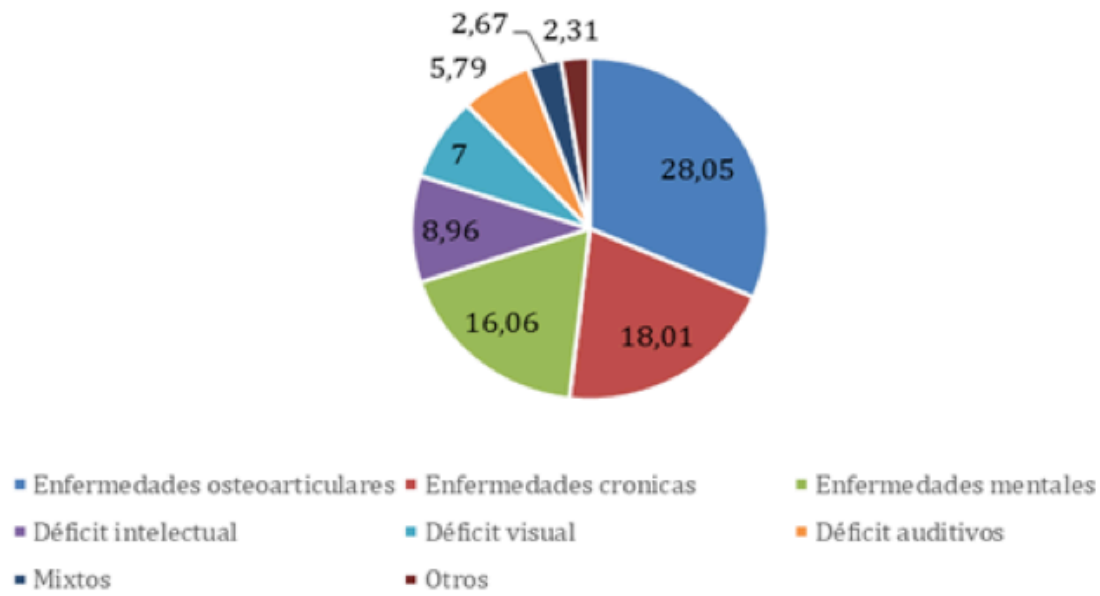

son el tercer grupo en cuanto a tamaño, como se puede observar en los datos de la encuesta EDAD (MSSS, 2008).

Cabe remarcar que, a nivel laboral, España ha implementado leyes que promueven la inclusión de personas con discapacidad en el mercado laboral, esto ha podido reducir la discapacidad de las personas debida a factores mentales. Sin embargo, las personas que sufren de trastornos mentales son percibidas frente a las personas que sufren discapacidades físicas y discapacidades sensoriales, con peor predisposición a la contratación por los empresarios, según un estudio realizado en una muestra de 1.503 empresas (Fundación, O.N.C.E., \& MANPOWER, FUNDATION. 2008). Esto muestra que, a pesar de que se ha avanzado mucho en la integración social y laboral de personas con discapacidad, todavía existen barreras importantes para que personas con depresión y otros trastornos mentales, los cuáles sufren elevados niveles de discapacidad.

Existen recientes estudios que muestran que, de todas las enfermedades mentales, neurológicas y por abuso de sustancias, la depresión es ya la primera causa de discapacidad (Whiteford, Ferrari, Degenhardt, Feigin, y Vos, 2015). En España, la tercera fuente de discapacidad está relacionada con problemas de salud mental, según la European Study of the Epidemiology of Mental Disorders (ESEMeD-España) (Haro et al., 2006). Por otra parte, según la OMS, los trastornos mentales comunes tienen consecuencias de gran envergadura sobre la pérdida de la salud. De estos, el TDM es el mayor contribuyente a la discapacidad. Los datos indican que un 7,5\% de todos los Años de Vida con Discapacidad (AVD) están relacionados con dicho trastorno, mientras que los trastornos de ansiedad ocupan el sexto 
Figura 3: Coste de los trastornos del cerebro. Fuente: Adaptado de Olesen et al. (2012)

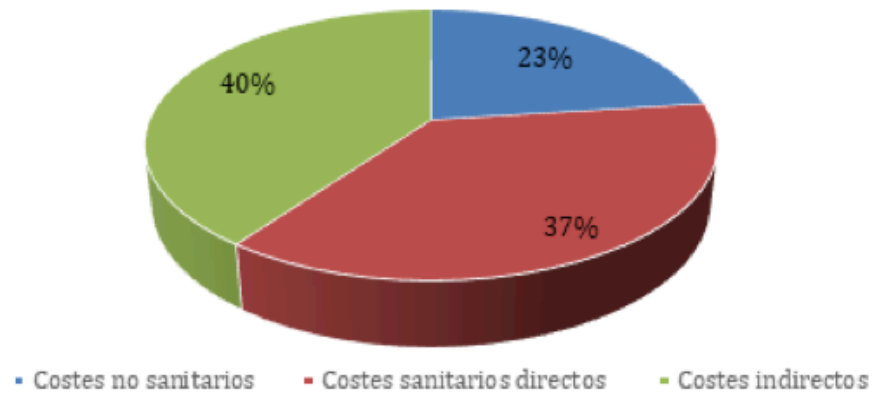

lugar (con un 3.4\% de los AVD). Además, la depresión es la enfermedad que más se relaciona con muertes por suicidio, los cuales ascienden a cerca de 800,000 al año en todo el mundo. Según este informe, España presenta una prevalencia de la depresión del 5.2\%, siendo el porcentaje de todos los AVD atribuidos a la depresión del $8.7 \%$ y para los trastornos de ansiedad el 3.6\%. Estas cifras destacan por ser superiores a la media mundial (WHO, 2017).

\section{Costes y depresión}

Los trastornos mentales son muy prevalentes, situándose alrededor de un $10 \%$ de prevalencia a nivel mundial (WHO, 2001). Generan un gran coste a la sociedad y, según la Organización Mundial de la Salud (OMS), serán los responsables de la mayor carga económica y social de todos los países en el año 2020 (OMS, 2001). Asimismo, algunos estudios predicen que tan solo uno de todos los trastornos mentales, la depresión, se convertirá en una de las primeras causas de discapacidad a nivel mundial en el 2030 (Mathers \& Loncar, 2006).

Según Olesen et al. (2012), los trastornos psicológicos, especialmente los relacionados con la ansiedad y la depresión general unos costes de 74.380 millones de Euros y 91.914 millones respectivamente. Estos trastornos se han ido convirtiendo y se convertirán en la mayor fuente de reducción de calidad de vida y aumento de la discapacidad de las sociedades occidentales. Su alta prevalencia y su impacto en la salud general se ha conformado como un grave problema de salud pública que requiere ser atendido de forma imperiosa, dado los costes económicos y sociales que generan. Tal y como muestra Olesen et al. (2012), se estimó que en el 2010 un tercio de los europeos sufrió un trastorno cerebral (enfermedades neurológicas y los trastornos mentales), supuso unos costes de 798 billones de euros (un promedio de 5.550 por habitante), de los que se destinaban 186 billones (un 23\%) a costes directos no sanitarios; 295 billones (un 37\%) a costes directos sanitarios y 315 billones (un $40 \%$ ) a costes indirectos (discapacidad crónica, bajas laborales, etc.; ver Figura 3).

Concretamente, en España, se ha evaluado recientemente el coste de estos problemas de salud. Los costes generales de los trastornos mentales y del cerebro en España (Parés-Badell 
et al., 2014) en el año 2010 alcanzaron los 83.749 millones de euros anuales (aproximadamente el $8 \%$ del PIB de nuestro país en 2010), lo que superó el gasto público sanitario total, que fue de 69.357 millones de euros (6,6\% del PIB de 2010). De acuerdo con los datos de este estudio, solo los trastornos mentales generaron un coste de 46.000 millones de euros al año (cerca del 4,6\% del Producto interior bruto español). De estos, los llamados trastornos emocionales, (trastornos depresivos, ansiedad y somatizaciones) alcanzarían un gasto total de 22.778 millones de euros (2,2\% del PIB), casi la mitad del coste de todos los trastornos mentales, debido entre otros factores a su alta prevalencia. Los trastornos depresivos supondrían 10.763 millones de euros (1\%), los de ansiedad $10.365(1 \%)$ y las somatizaciones $1.650(0,2 \%)$ (Parés-Badell et al., 2014). Estos datos muestran la envergadura del problema al que nos enfrentamos.

Frente a esta situación, la OMS ha recomendado movilizar recursos que permitan dispensar las intervenciones adecuadas dentro de los sistemas de salud. En esta línea, el avance y desarrollo científico ha permitido el hallazgo de tratamientos y técnicas cada vez más eficaces para tratar tanto las patologías físicas como las psicológicas, aumentando así la esperanza y calidad de vida en aquellos países en los que la población tiene acceso a los recursos necesarios para cuidar su salud, fundamentalmente gracias a los sistemas sanitarios públicos. No obstante, los recursos destinados al ámbito de salud, un $8.7 \%$ del Producto Interior Bruto (PIB) de media a nivel global (WHO, 2009) comparado con el gasto destinado a las enfermedades relacionadas con problemas de salud mental no ha sido proporcional al nivel de discapacidad y prevalencia que estos trastornos presentan. Esta baja inversión y, por tanto, el bajo acceso a tratamientos mínimamente adecuados genera un coste por problemas de salud mental en los países desarrollados de entre el $3 \%$ y el 4\% del PIB (WHO, 2004). Mientras, el porcentaje destinado a paliar este tipo de patología es únicamente $3 \%$ de las partidas correspondientes a salud (Cortés, 2010).

\section{Depresión y otros trastornos emocionales en atención primaria.}

La mayoría de las personas con este trastorno psicológico acuden a solicitar ayuda en los servicios de Atención Primaria, la puerta de entrada al sistema de salud. Según Haro et al. (2006), aproximadamente 1 de cada 3 pacientes que acuden a los servicios de AP sufre de algún tipo de trastorno mental. Por otra parte, según Roca et al. (2009), el 49,2\% de los pacientes del territorio español que acuden a las consultas de Atención Primaria cumplen los criterios diagnósticos para al menos un trastorno de ansiedad, depresión o somatización. Esto muestra la elevada comorbilidad de la depresión con otros trastornos emocionales, la cuál está estrechamente relacionada con la mala calidad de vida, abuso de sustancias, discapacidad y altos costos sociales y sanitarios. Además, estos últimos aumentan, además, en paralelo con el incremento en el número de trastornos comórbidos. Así que la depresión y otros trastornos emocionales no solo son una de las principales causas de discapacidad sino también de demanda de servicios de Atención Primaria presentando una prevalencia en AP que oscila entre el 9,6\% y el 20,2\% (Cano Vindel et al., 2012). Con ello, podemos observar que estos trastornos, en caso de recibir un tratamiento inadecuado o sin resultados, los pacientes pueden generar consecuencias muy negativas como reducción de la calidad de vida (Spitzer et al., 1995), la cronificación de problemas emocionales (Kessler et al., 2011), el in- 
cremento del uso de los servicios de salud (servicios de atención primaria, médico general, hospitales, etc.) (Greenberg, Stiglin, Finkelstein, \& Berndt, 1993), o el aumento del riesgo de suicidio (Oquendo et al., 2002).

Esta prevalencia está muy relacionada con situaciones sociales derivadas del contexto de crisis que hemos vivido. De hecho, el estudio IMPACT, que comparó la prevalencia de problemas de salud mental en AP antes y durante la crisis (Gili et al. 2014) mostró un incremento significativo de las alteraciones del estado de ánimo específicamente para el año 2006 y 2010 , en el caso de la depresión mayor las tasas no ajustadas de prevalencia fueron de $28.9 \%$ y $47,5 \%$; en el caso de la depresión menor fueron de $6,4 \%$ y $47.5 \%$ y; para la distimia de un $14,6 \%$ y $25,1 \%$ (Gili et al., 2013). Esto representa un aumento del $19,4 \%$ en el trastorno depresivo mayor. Para otros trastornos los incrementos durante dicha etapa fueron del 10,8\% para la distimia, el 8,4\% para el trastorno de ansiedad generalizada; el 7,2 \% para los trastornos somatomorfos; el 6,4 \% para las crisis de angustia; un 4,6\% para la dependencia de alcohol y finalmente; un 2,4\% para el abuso de alcohol. Además, existen diversos factores que posicionan actualmente la depresión como una de las principales causas de discapacidad, representando un $4,3 \%$ de la carga global de enfermedad como, por ejemplo, la tendencia a la cronicidad y recurrencia, su inicio temprano o el impacto que tiene, a nivel funcional, en la vida de los sujetos que la padecen. (Álvarez Ariza, 2014). De forma recíproca, Kessler et al. (2003) también pone en evidencia el desajuste que la depresión puede ocasionar en áreas como el trabajo, las relaciones sociales e íntimas y las actividades cotidianas, por lo que la espiral de discapacidad, depresión y baja productividad se intensifica.

Sabiendo lo anterior, hallamos otros dos problemas que impiden que los profesionales de AP puedan realizar una buena labor que se traduzca en una gestión de los recursos sanitarios eficiente. Por una parte, nos encontramos que la concordancia entre los diagnósticos de AP y los emitidos en salud mental difieren según el tipo de trastornos, mermando esto tanto la calidad de la atención recibida por los pacientes como la eficiencia en el uso de los recursos disponibles. Algunos autores como González et al. (2008) señalan las diferencias en dicha concordancia: mientras que para los trastornos como el uso de alcohol y sustancias o los trastornos psicóticos es buena (kappa > 0,7) o para los Trastornos de la Conducta Alimentaria o los trastornos mentales orgánicos es moderada (índice kappa entre 0,4 y 0,7 ), para los trastornos que, como comentábamos anteriormente suponen casi la mitad del coste de todos los trastornos mentales, nos encontramos una concordancia baja (kappa $<0,3)$. Precisamente los grupos de trastornos que mayores costes generan son los que cuentan con menor concordancia (los afectivos obtienen un valor de kappa de 0,18 , seguidos por los ansiosos, con un índice kappa de 0,23). Así mismo, un meta-análisis con 41 estudios que comprendían 50.371 pacientes encontró que la depresión no llegaba a ser bien diagnosticada ni en la mitad de los casos (solo en un 47,3\%) (Mitchel et al., 2009).

\section{Discusión}

En este trabajo se ha observado que la depresión, junto con otros trastornos comórbidos es muy prevalente y genera una elevada discapacidad. Esta discapacidad, a su vez, genera costes económicos enormes, entre costes directos e indirectos. Los pacientes que sufren 
esta patología acuden en primer lugar a las consultas de AP, donde no solo hay errores de diagnóstico, si no también, de intervención. Por tanto, es necesario mejorar la organización de AP que permita hacer frente a esta grave problemática.

Tras nuestra revisión se observa que la depresión es altamente prevalente y no se está dando respuesta a su impacto. Todo esto se deduce por datos como los que muestra la OMS (2001), que calcula que hay una prevalencia entorno al 10\% de trastornos mentales a nivel mundial, los recursos destinados a salud son del $8.7 \%$ del PIB de media global, siendo específicamente los destinados a salud mental el 0,261 del PIB (Cortés, 2010). Así, estos trastornos generan un elevado coste económico, donde trastornos mentales generaron el equivalente al 4,6\% del PIB. De éstos, los trastornos emocionales (depresivos, de ansiedad y por somatizaciones) alcanzaron un total de 22.778 millones de euros $(2,2 \%$ del PIB) y de éstos, solo los trastornos depresivos supondrían 10.763 millones de euros (1\% del PIB) (Parés-Badell et al., 2014).

En España, encontramos que los trastornos emocionales son una de las principales causas de dependencia (la tercera según Haro et al., 2006) dado que un 16,06\% de las personas con una dependencia superior al $33 \%$ entran en este colectivo debido a estos trastornos mentales comunes (MSSS, 2008), generando, además de la discapacidad, una alta demanda en Atención Primaria (Cano-Vindel et al., 2016). De todos ellos, se califica el TDM como el mayor contribuyente a la discapacidad (un 7,5\% de media mundial y un 8,7\% en España) de todos los AVD (OMS, 2017), representando un 4,3\% de la carga global de enfermedad (Álvarez Ariza, 2014). A la alta prevalencia (Mitchell, Vaze, \& Rao, 2009; Roca et al. 2009) se le suma que el diagnóstico en atención primaria, que reduciría la sobrecarga asistencial del sistema de salud, no suele ser concordante con los diagnósticos de los especialistas (González et al. 2008) y esto impide la derivación a tratamientos más recomendados (Mitchel et al., 2009) siendo, además, el tratamiento habitual farmacológico menos adecuado que los tratamientos psicológicos (Watts et al., 2015).

Las consultas de AP, como puerta de entrada al sistema sanitario público, tienen una elevada demanda de este tipo de patologías, cerca del 49,2\% (Roca et al., 2009). Por tanto, se debe mejorar los procesos de intervención en este ámbito. En primer lugar, es prioritario poner el foco en un buen diagnóstico de los trastornos emocionales, especialmente en los trastornos depresivos pues no siempre son bien diagnosticadas lo cuál genera importantes consecuencias negativas (cronificación, incremento de uso de los recursos sociosanitarios, decremento en la calidad de vida, riesgo de suicidio, etc; Cano Vindel et al., 2012). Un modelo que reúne las características mencionadas y subsana las problemáticas del tratamiento habitual en AP, es el modelo jerárquico de cribado, diagnóstico y derivación de los trastornos mentales en centros de atención primaria que se deriva del ensayo clínico PsicAP (Muñoz-Navarro et al. 2017). Dicho modelo propone 3 escalones de detección y diagnóstico. En primer lugar, pone a disposición de médico de AP una versión de cribado ultracorta derivada del PHQ, el PHQ-4 (Cano-Vindel et al., 2018). En caso de dar positivo, se procede a la derivación al psicólogo clínico, el cual haría un cribado con el PHQ completo. Finalmente, en psicólogo clínico realizaría una entrevista clínica, donde confirmaría el diagnóstico y tomaría una decisión de derivación a tratamiento en AP o atención especializada. En caso de que el nivel de depresión fuera moderado o leve, el tratamiento se efectúa en AP. 
Por otra parte, además de un buen diagnóstico, es fundamental para el paciente que acude a AP una buena intervención. En referencia a los tratamientos para los trastornos depresivos, aunque los tratamientos farmacológicos son los más utilizados y fueron los primeros en aplicarse (Pérez-Alvárez y García-Montes, 2001), no son los más adecuados (Watts et al., 2015). Existen tres modalidades de tratamientos psicológicos que cuentan con suficiente evidencia empírica para denominarlos bien establecidos, estos son, según los autores arriba citados, la terapia de conducta en la cual incluimos: el tratamiento mediante programa de actividades agradables; entrenamiento en habilidades sociales (EHS); el programa de Lewinshon para el afrontamiento de la depresión; la terapia de autocontrol; la terapia de resolución de problemas y; en casos donde además haya problemas en la relación de pareja, la terapia conductual de pareja. También se incluyen, de corte más cognitivo, la terapia para la depresión de Beck y la psicoterapia interpersonal desarrollada por Klerman y Weissman. Para mejorar la efectividad de los tratamientos también es necesario que los terapeutas encargados de implementarlo tengan formación específica y estén entrenados, que los propios tratamientos cuenten con una guía y se dirijan a componentes específicos (Cano Vindel et al., 2012).

\section{Conclusiones}

A lo largo del texto se ha podido fundamentar la necesidad de implantación de un modelo alternativo al tratamiento habitual en general de los trastornos mentales más prevalentes y específicamente de la depresión. Los datos presentados muestran varios focos donde se puede mejorar la eficiencia ante los que una actuación basada en la evidencia empírica devengará en beneficios para la sociedad, reduciendo la presencia de trastornos emocionales y con ello, los costes directos e indirectos asociados a ellos.

Más allá de las necesarias consideraciones empíricas, se debe tener en cuenta un aspecto importante: el derecho del paciente a decidir sobre su tratamiento. McHugh, Whitton, Peckham, Welge y Otto (2013), en un metaanálisis, obtuvieron como resultado que los pacientes prefieren tres veces más un tratamiento psicológico frente al farmacológico. La posibilidad de ofrecer esta intervención a los pacientes con trastornos depresivos, de ansiedad y/o somatizaciones en atención primaria incluye atender a las necesidades expresadas por los mismos, así como seguir las recomendaciones de la NICE en cuanto a recomendaciones de tratamiento de dichos trastornos.

\section{BIBLIOGRAFÍA}

Álvarez Ariza, M., Atienza Merino, G., Avila González, M. J., González García, A., Guitian Rodríguez, D., Louro González, A., \& Triñares Pego, Y. (2014). Guía de Práctica Clínica sobre el Manejo de la Depresión en el Adulto. Ministerio de Sanidad, Servicios Sociales e Igualdad. Agencia de Evaluación de Tecnologías Sanitarias de Galicia, avalia-t.

American Psychiatric Association. (2000). Diagnostic and statistical manual of mental disorders DSM-IV-TR fourth edition (text revision). American Psychiatric Association.

American Psychiatric Association (APA). (2013). DSM-5. Diagnostic and statistical manual of mental disorders (5th ed.). Washington, DC. 
Cano-Vindel, A., Muñoz-Navarro, R., Wood, C. M., Limonero, J. T., Medrano, L. A., Ruiz-Rodríguez, P., y Santolaya, F. (2016). Transdiagnostic cognitive behavioral therapy versus treatment as usual in adult patients with emotional disorders in the primary care setting (PsicAP study): protocol for a randomized controlled trial. JMIR Research Protocols, 5(4).

Cano-Vindel, A., Salguero, J. M., Mae Wood, C., Dongil, E., \& Latorre, J. M. (2012). La depresión en atención primaria: prevalencia, diagnóstico y tratamiento. Papeles del psicólogo, 33(1).

Cortés, M. P. (2010). Economía de la salud mental. El Residente, 5(1), 9-13.

Greenberg, P. E., Stiglin, L. E., Finkelstein, S. N., \& Berndt, E. R. (1993). Depression: a neglected major illness. Journal of Clinical Psychiatry, 54, 419-24.

Esparza Catalán, C. (2011). Discapacidad y dependencia en España. Madrid, Informes Portal Mayores, 108. Disponible en: http://www.imsersomayores.csic.es/documentos/pm-discapacidad-01.pdf

Fundación, O.N.C.E., \& MANPOWER, F. (2008). Perspectivas del mundo empresarial, respecto a la contratación de personas con discapacidad. [Informe]. Madrid.

Gili, M., Roca, M., Basu, S., McKee, M., \& Stuckler, D. (2013). The mental health risks of economic crisis in Spain: evidence from primary care centres, 2006 and 2010. The European Journal of Public Health, 23(1), 103-108.

Gili, M., Campayo, J. G., \& Roca, M. (2014). Crisis económica y salud mental. Informe SESPAS 2014. Gaceta Sanitaria, 28, 104-108.

González, N. L., Sarriés, A. G., de Jalón Aramayo, E. G., \& Urra, E. L. (2008). Concordancia en el diagnóstico entre atención primaria y salud mental. Atención primaria, 40(6), 285-289.

González-Blanch, C., Umaran-Alfageme, 0., Cordero-Andrés, P., Muñoz-Navarro, R., Ruiz-Rodríguez, P., Medrano, L. A., \& Cano-Vindel, A. (2017). Tratamiento psicológico de los trastornos emocionales en Atención Primaria: el manual de tratamiento transdiagnóstico del estudio PsicAP. Ansiedad y Estrés, 23 (2-3), 91-98.

Haro, J. M., Palacín, C., Vilagut, G., Martínez, M., Bernal, M., Luque, I., \& Alonso, J. (2006). Prevalencia de los trastornos mentales y factores asociados: resultados del estudio ESEMeD-España. Medicina Clínica, 126(12), 445-451.

IMSERSO (2016). Base estatal de datos de personas con valoración del grado de discapacidad. Madrid: subdirección general de planificación, ordenación y evaluación. Disponible en : http://www.imserso.es/InterPresent1/groups/imserso/documents/binario/bdepcd_2015.pdf

INE. (2018). Encuesta sobre discapacidades, autonomía personal y situaciones de dependencia. Madrid: Instituto Nacional de Estadística. Disponible en: http://www.ine.es/dyngs/INEbase/es/operacion.htm?c=Esta distica_C\&cid=1254736176782\&menu=resultados\&secc=1254736194716\&idp=1254735573175\#

Kessler, R. C., Berglund, P., Demler, 0., Jin, R., Koretz, D., Merikangas, K. R., Wang, P. S. (2003). The epidemiology of major depressive disorder: results from the National Comorbidity Survey Replication (NCS-R). Journal of the American Medical Association, 289, 3095-3105.

Kessler, R. C., Ormel, J., Petukhova, M., McLaughlin, K.A., Green, J. G., Russo, L. J., Ustun, T. B. (2011). Development of lifetime comorbidity in the World Health Organization world mental health surveys. Archives of General Psychiatry, 68, 90-100.

Mathers, C. D., \& Loncar, D. (2006). Projections of global mortality and burden of disease from 2002 to 2030. PLoSMedicine, 3(11), e442. doi:10.1371/journal.pmed.0030442.g009

McHugh, R. K., Whitton, S. W., Peckham, A. D., Welge, J. A., \& Otto, M. W. (2013). Patient preference for psychological vs. pharmacological treatment of psychiatric disorders: a meta-analytic review. The Journal of Clinical Psychiatry, 74(6), 595.

Ministerio de Sanidad, Servicios Sociales e Igualdad (2015). Utilización de medicamentos antidepresivos en España durante el periodo 2000-2013. Disponible en http://www.aemps.gob.es/medicamentosUsoHumano/observatorio/ docs/antidepresivos-2000-2013.pdf

Mukuria, C., Brazier, J., Barkham, M., Connell, J., Hardy, G., Hutten, R., \& Parry, G. (2013). Cost-effectiveness of an improving access to psychological therapies service. The British Journal of Psychiatry, 202(3), 220-227.

Muñoz-Navarro, R., Cano-Vindel, A., Ruiz-Rodríguez, P., Medrano, L. A., González-Blanch, C., Moriana, J. A. y DongilCollado, E. (2017). Modelo jerárquico de diagnóstico y derivación de los trastornos mentales comunes en centros de atención primaria. Una propuesta a partir del ensayo clínico PsicAP. Ansiedad y Estrés; 23:124-9. 
Oquendo, M. A., Halberstam, B., \& Mann, J. J. (2003). Risk factors for suicidal behavior. Standardized evaluation in clinical practice, 22, 103-129.

Olesen, $\mathrm{J}$ et al. (2012). The economic cost of brain disorders in Europe. European Journal of Neurology; 19: 155-162.

Parés-Badell 0, et al. (2014). Cost of Disorders of the Brain in Spain. PLos ONE, 9(8): e105471. doi:10.1371/journal. pone. 0105471 .

Paykel, E.S., Brugha, T., \& Fryers, T. (2005). Size and burden of depressive disorders in Europe. European Neuropsychopharmacology, 5, 411-423.

Pérez-Alvárez, M., y García-Montes, J. M. (2001). Tratamientos psicológicos eficaces para la depresión. Psicothema, 13(3).

Roca, M., Gili, M., Garcia-Garcia, M., Salva, J., Vives, M., Garcia Campayo, J., \& Comas, A. (2009). Prevalence and comorbidity of common mental disorders in primary care. Journal of Affective Disorders, 119(1), 52-58. doi:10.1016/j.jad.2009.03.014

Ruiz-Rodríguez, P., Cano-Vindel, A., Navarro, R. M., Medrano, L., Moriana, J. A., Aguado, C. B., \& González-Blanch, C. (2017). Impacto económico y carga de los trastornos mentales comunes en España: una revisión sistemática y crítica. Ansiedad y Estrés, 23 (2-3), 118-123.

Santos-Ruiz, J. y Hernan-gómez Criado, L. (2014). Psicopatología. Madrid: CEDE.

Santos Ruiz, J., Sanz Rodríguez, L. y Álvarez Calderón, M. (2016). Psicología clínica. 3rd ed. Madrid: CEDE, p.243.

Santos-Ruiz, J., Sanz-Rodríguez, L. y Álvarez-Calderón, M. (2014). Psicología clínica. Madrid: CEDE.

Spitzer, R. L., Kroenke, K., Linzer, M., Hahn, S. R., Williams, J. B., deGruy, F. V., Davies, M. (1995). Health-related quality of life in primary care patients with mental disorders. Results from the PRI-MEMD 1000 Study. JAMA, 274, $1511-1517$

Watts, S. E., Turnell, A., Kladnitski, N., Newby, J. M., \& Andrews, G. (2015). Treatment-as-usual (TAU) is anything but usual: a meta-analysis of CBT versus TAU for anxiety and depression. Journal of affective disorders, 175, 152-167

Whiteford, H. A., Ferrari, A. J., Degenhardt, L., Feigin, V., \& Vos, T. (2015). The global burden of mental, neurological and substance use disorders: an analysis from the Global Burden of Disease Study 2010. PloS one, 10(2), e0116820.

World Health Organization. (2001). The world health report: Mental health: New understanding, new hope. Geneva, Switzerland. WHO.

World Health Organization. (2004). Invertir en salud mental. Geneva, Switzerland. WHO.

World Health Organization. (2009). Estadísticas sanitarias mundiales 2009. Geneva, Switzerland. WHO.

World Health Organization. (2017). Depression and other common mental disorders: Global health estimates. Geneva: World Health Organization. Disponible en: http://www.who.int/mental health/management/depression/prevalence_global_health_estimates/en/ 